\title{
Do Surgical Margins Affect Local Recurrence and Survival in Extremity, Nonmetastatic, High-grade Osteosarcoma?
}

\author{
Todd E. Bertrand MD, Alex Cruz BS, Odion Binitie MD, \\ David Cheong MD, G. Douglas Letson MD
}

Published online: 27 May 2015

(C) The Association of Bone and Joint Surgeons ( 2015

\begin{abstract}
Background Long-term survival for all patients with osteosarcoma using current aggressive adjuvant chemotherapy and surgical resection is between $60 \%$ and $70 \%$. In patients who present with nonmetastatic, high-grade extremity osteosarcoma of bone, limb salvage surgery is favored, when appropriate, over amputation to preserve the limb, because limb salvage may lead to a superior quality of life compared with amputation. However, concern remains that in the attempt to preserve the limb, close or microscopically positive
\end{abstract}

Each author certifies that he or she, or a member of his or her immediate family, has no funding or commercial associations (eg, consultancies, stock ownership, equity interest, patent/licensing arrangements, etc) that might pose a conflict of interest in connection with the submitted article.

All ICMJE Conflict of Interest Forms for authors and Clinical Orthopaedics and Related Research ${ }^{\mathbb{R}}$ editors and board members are on file with the publication and can be viewed on request.

Each author certifies that his or her institution approved the human protocol for this investigation, that all investigations were conducted in conformity with ethical principles of research, and that informed consent for participation in the study was obtained.

This work was performed at H. Lee Moffitt Cancer Center and Research Institute, Tampa, FL, USA.

T. E. Bertrand, O. Binitie, D. Cheong, G. D. Letson

Department of Sarcoma, H. Lee Moffitt Cancer Center and

Research Institute, Tampa, FL, USA

\section{A. Cruz}

University of South Florida School of Medicine, Tampa, FL, USA

T. E. Bertrand $(\bowtie)$

IU Health University Hospital, 550 N University Boulevard, Suite 1295, Indianapolis, IN 46202, USA

e-mail: tbertrand@iuhealth.org surgical margins may have an adverse effect on event-free survival.

Questions/purposes (1) Does a positive or close surgical margin increase the likelihood of a local recurrence? (2) Does a positive or close surgical margin adversely affect the development of metastatic disease? (3) What is the relationship of surgical margin on overall survival?

Methods With institutional review board approval, we retrospectively evaluated 241 patients treated at our institution between 1999 and 2011. Exclusion criteria included nonextremity locations, metastatic disease at initial presentation, low- or intermediate-grade osteosarcoma, treatment regimens that did not follow National Comprehensive Cancer Network (NCCN) guidelines, incomplete medical records, and any part of treatment performed outside of Moffitt Cancer Center or All Children's Hospital. Fifty-one patients were included in the final analysis, of whom 31 (61\%) had followup data at a minimum of 2 years or whose clinical status was known but had died before 2 years of followup. Margin status was defined as (1) microscopically positive; (2) negative $\leq 1 \mathrm{~mm}$; and (3) negative $>1 \mathrm{~mm}$. Margin status, histologic response (tumor percent necrosis), type of osteosarcoma, type of surgery, presence of local recurrence, metastatic disease, and overall survival were recorded for each patient. The mean age was 22 years (range, 12-74 years) and the mean followup was 3 years (range, 0.1-14 years). Margin status was positive in $10 \%$ (five of 51), negative $\leq 1 \mathrm{~mm} \mathrm{26 \%} \mathrm{(13} \mathrm{of} \mathrm{51),} \mathrm{and} \mathrm{negative}$ $>1 \mathrm{~mm} 65 \%$ (33 of 51).

Results Local recurrence was noted to be $14 \%$ (seven of 51) at 3.4 years. After controlling for relevant confounding variables, the presence of a positive margin compared with a negative margin $>1 \mathrm{~mm}$ was the only independent predictor of local recurrence (hazard ratio [HR], 8.006; $95 \%$ confidence interval [CI], 1.314-48.781; $\mathrm{p}=0.0241)$. At a mean of 3.4 
years, $29 \%$ (15 of 51 ) of the patients developed metastatic disease with no difference with the numbers available in the probability of developing metastatic disease among the three margin groups $(\mathrm{p}=0.614)$. Overall survival at 3.8 years was $75 \%$ (38 of 51). After controlling for relevant confounding variables, we found that patients with positive margins were more likely to die from disease than those with negative margins (HR, 6.26; 95\% CI, 1.50-26.14; $\mathrm{p}=0.0119$ ); no other independent predictors of survival were identified.

Conclusions With the numbers of patients we had, we observed that patients with extremity, nonmetastatic, highgrade osteosarcoma who had positive margins showed a higher probability of local recurrence in comparison to those with negative surgical margins. Given that positive margins appear to be associated with poorer survival in patients with high-grade osteosarcoma of the extremities, surgeons should strive to achieve negative margins, but larger studies are needed to confirm these findings. Level of Evidence Level III, therapeutic study.

\section{Introduction}

Overall survival in all patients with osteosarcoma using currently accepted treatment modalities of neoadjuvant chemotherapy, local surgical control, and adjuvant chemotherapy is approximately $60 \%$ to $70 \%[4,11]$. Limb preservation is preferred over amputation when possible for local control because this may allow for increased function and improved quality of life.

However, in the context of limb preservation surgery, inadequate surgical margins have been associated with an increased incidence of local recurrence in osteosarcoma, and the smallest safe margin width still remains controversial. Because local recurrence may adversely affect survival, this is a key issue when considering limb-sparing resections. In one study enrolling 837 patients with a combination of low- and highgrade bone and soft tissue sarcomas, an adequate margin was found to be $2 \mathrm{~cm}$ when the preoperative treatment modality (chemotherapy or radiation) was effective as determined by a marked decrease in size of the tumor on posttreatment imaging before surgical resection. Histopathological correlation between tumor percent necrosis and local recurrence was not done [9]. However, in a second study involving patients with high-grade osteosarcoma that was nonmetastatic at presentation, there was no difference in local recurrence between margins less than or equal to $2 \mathrm{~mm}$ versus more than $2 \mathrm{~mm}$ [3].

With institutional review board approval we therefore retrospectively studied our experience in patients with extremity, nonmetastatic, high-grade osteosarcoma in terms of the impact of margin status on oncologic outcomes. Specifically, we asked the following questions: (1) Does a positive or close surgical margin increase the likelihood of a local recurrence? (2) Does a positive or close surgical margin adversely affect the development of metastatic disease? (3) What is the relationship of surgical margin on overall survival?

\section{Patients and Methods}

\section{Demographics}

From 1999 to 2011, we treated 241 patients at our institution for osteosarcoma. We excluded 90 (37\%) patients for incomplete medical records, 34 (14\%) patients whose primary site of disease was extraosseous or in a nonextremity location, 35 (15\%) patients in whom some of their treatment was performed outside of H. Lee Moffitt Cancer Center and All Children's Hospital, 16 (7\%) patients whose treatment did not follow National Comprehensive Cancer Network guidelines, seven (3\%) patients whose disease was of osseous origin in an extremity but not a long bone (ie, hands and feet), five (2\%) patients who had metastatic disease at presentation, and three $(1 \%)$ patients who had low- or intermediate-grade histologies. Patients who had died or reached another clinical endpoint before 2 years and whose status was known were included. This left 51 (21\%) patients who satisfied the prespecified study inclusion and exclusion criteria (Fig. 1). All diagnoses were confirmed histologically.

There were a total of $20(39 \%)$ patients who had clinical followup of less than 2 years. Of these, $16(80 \%)$ were lost to followup and four (20\%) were secondary to death during the followup period. All patients who were lost to death before 2 years from surgery had positive margins. Of the remaining 16 patients, five $(31 \%)$ had close margins of 1 $\mathrm{mm}$ or less. There were no patients lost to followup, aside from death, with positive margins. There were $31(61 \%)$ patients with a minimum followup of 2 years from the time of surgery. Of these, eight (26\%) had close margins of 1 $\mathrm{mm}$ or less and two $(7 \%)$ had positive margins (Table 1).

After biopsy all patients underwent two cycles of neoadjuvant chemotherapy with a three-drug regimen of Adriamycin, cisplatin, and high-dose methotrexate [6]. After surgical wound healing from local control, patients underwent between zero and four cycles of adjuvant chemotherapy consisting of the same three-drug regimen in Cycles 3 and 4 and only Adriamycin with high-dose methotrexate in Cycles 5 and 6. The determination for adjuvant chemotherapy was multifactorial and dependent on factors such as patient age, histological response of the tumor to the neoadjuvant treatment, and the discretion of the medical and orthopaedic oncologists. 

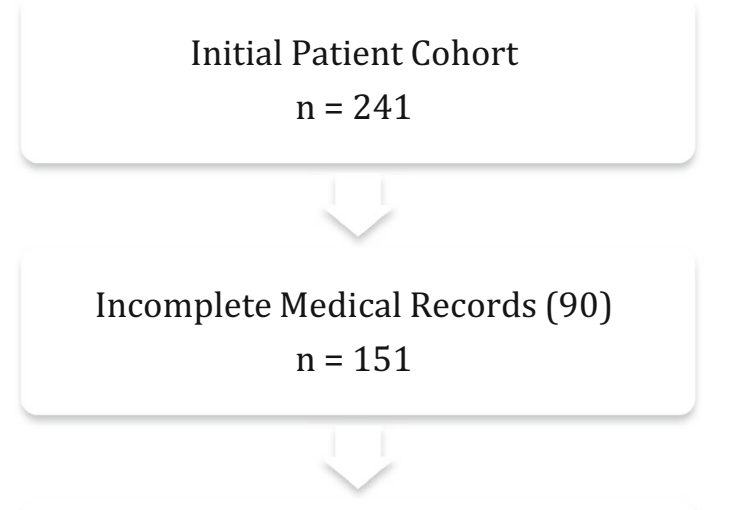

Nonextremity Locations (34)

$\mathrm{n}=117$
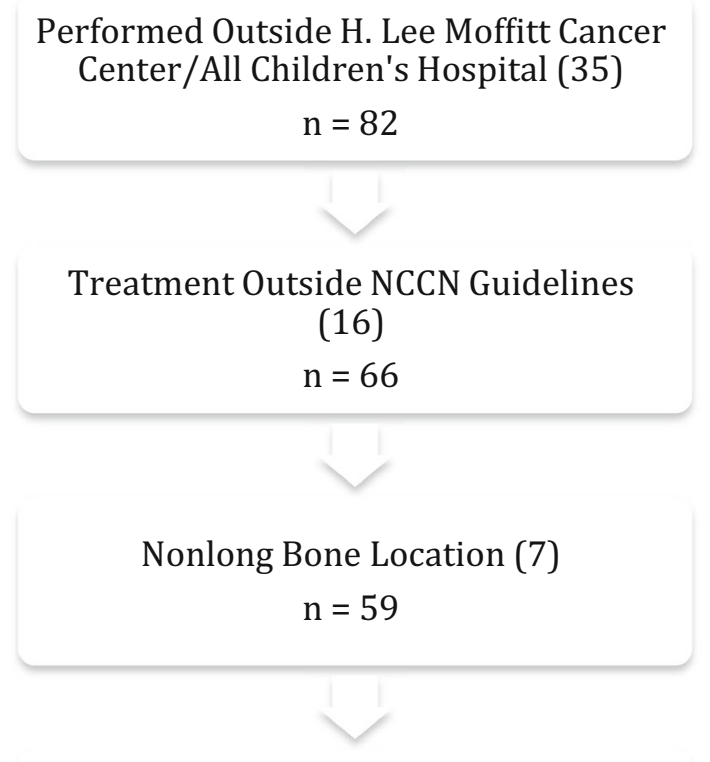

Metastatic Disease (5)

$n=54$

Low/Intermediate Grade (3)

$\mathrm{n}=51$

Fig. 1 The flowchart shows exclusion criteria for our patient cohort.
Table 1. Comparison between patients with less than and minimum 2 years followup

\begin{tabular}{lll}
\hline Followup period & $\begin{array}{l}\text { Minimum } \\
\text { followup 2 years }\end{array}$ & $\begin{array}{l}\text { Followup less than } \\
\text { 2 years (excluding death) }\end{array}$ \\
\hline Total number & 31 & 16 \\
Positive margins & $2(7 \%)$ & $0(0 \%)$ \\
Margins $\leq 1 \mathrm{~mm}$ & $8(26 \%)$ & $5(31 \%)$ \\
Local recurrence & $6(19 \%)$ & $1(6 \%)$ \\
Metastatic disease & $9(29 \%)$ & $6(38 \%)$ \\
\hline
\end{tabular}

Definitive surgery for local control was performed within 4 weeks of the completion of neoadjuvant chemotherapy. Forty-six patients underwent limb preservation surgery $(90 \%)$ and five patients underwent amputation (10\%) including three forequarter amputations and two above-knee amputations. General indications for amputation in our patient cohort included major vascular structures encased by tumor not amendable to vascular bypass, extensive soft tissue involvement, or severe infection within the surgical field that would have compromised limb reconstruction.

In five patients with a microscopically positive margin, three underwent reresection at the time of the initial surgical procedure and two did not. Reresection at the time of the initial operation was based on intraoperative frozen section from a concerning area. Those who did not undergo reresection did not have intraoperative frozen sections because it was felt at the time of surgery that the initial resection was adequate for achieving negative surgical margins. Postoperative radiation therapy was not given for a microscopic positive margin. All positive margins in our study were adjacent at an extraosseous soft tissue component of the tumor. For purposes of statistical analysis, all positive margins, whether determined intraoperatively with frozen section or at the time of final pathology, were grouped into the positive surgical margin group.

Patients were followed with both imaging and physical examination every 3 months for the first 2 years after surgery, every 6 months in the third year, and yearly in Years 4 and 5. After this time followup duration was determined at the discretion of the treating physician. Imaging included plain radiographs and MRI with contrast of the original tumor site as well as CT scan of the chest.

The attending pathologist (CMC, MB) determined the final margin status. For the purposes of our study, margin status was determined to be microscopically positive, negative $\leq 1 \mathrm{~mm}$, or negative $>1 \mathrm{~mm}$. There were no grossly positive resections in our patient cohort. All specimens were oriented and inked by the pathologist after review with the operative surgeon. A microscopically positive margin was reported when the tumor was present 
Table 2. Listing of study variables

\begin{tabular}{|c|c|c|c|}
\hline Variable & Level & Frequency & Percent \\
\hline \multirow[t]{3}{*}{ Margin (grouped) } & Negative margins $>1 \mathrm{~mm}$ & 33 & 65 \\
\hline & Negative margins $\leq 1 \mathrm{~mm}$ & 13 & 26 \\
\hline & Positive margins & 5 & 10 \\
\hline \multirow[t]{2}{*}{ Tumor percent necrosis } & $\leq 90 \%$ & 28 & 57 \\
\hline & $>90 \%$ & 21 & 43 \\
\hline \multirow[t]{7}{*}{ Type of osteosarcoma } & Chondroblastic & 11 & 22 \\
\hline & Fibroblastic & 1 & 2 \\
\hline & Giant cell rich & 2 & 4 \\
\hline & Osteoblastic & 31 & 61 \\
\hline & Periosteal & 2 & 4 \\
\hline & Small cell & 1 & 2 \\
\hline & Telangiectatic & 3 & 6 \\
\hline \multirow[t]{2}{*}{ Amputation or limb salvage } & Amputation & 5 & 10 \\
\hline & Limb salvage & 46 & 90 \\
\hline \multirow[t]{2}{*}{ Vital status } & Alive & 38 & 75 \\
\hline & Dead & 13 & 25 \\
\hline \multirow[t]{2}{*}{ Local recurrence status } & Local recurrence & 7 & 14 \\
\hline & No local recurrence & 44 & 86 \\
\hline \multirow[t]{2}{*}{ Metastasis status } & Metastasis & 15 & 29 \\
\hline & No metastasis & 36 & 71 \\
\hline
\end{tabular}

Table 3. Demographics of study population

\begin{tabular}{llll}
\hline Demographic & $\begin{array}{l}\text { Positive } \\
\text { margins }\end{array}$ & $\begin{array}{l}\text { Negative margins } \\
>1 \mathrm{~mm}\end{array}$ & $\begin{array}{l}\text { Negative margins } \\
\leq 1 \mathrm{~mm}\end{array}$ \\
\hline $\begin{array}{l}\text { Age at diagnosis } \\
\quad \text { years) }\end{array}$ & 28 & 22 & 21 \\
Side & & & \\
Left & 2 & 24 & 5 \\
Right & 3 & 9 & 8 \\
Sex & & & \\
Male & 2 & 19 & 5 \\
Female & 3 & 14 & 8 \\
Location & & & 2 \\
Upper extremity & 0 & 9 & 11 \\
$\quad$ Lower extremity & 5 & 24 & 45 \\
$\begin{array}{l}\text { Followup (months) } \\
\text { Size in largest }\end{array}$ & 23 & 45 & 9 \\
dimension (cm) & 12 & 10 & \\
\hline
\end{tabular}

on the inked margin. Surgical margins were positive in five of 51 patients $(10 \%)$, negative $\leq 1 \mathrm{~mm}$ in 13 of $51(26 \%)$, and negative $>1 \mathrm{~mm}$ in 33 of $51(65 \%)$.

When the margin was negative, the distance from the tumor to the closest margin was measured.

Margin status, histologic response (percent tumor necrosis), type of osteosarcoma, type of local control surgery, presence of local recurrence, metastatic disease, and overall survival were recorded for each patient (Table 2). Tumor percent necrosis was determined using the system developed by Huvos [8] by comparing the biopsy and resected specimen to see the degree of necrosis present. For two patients the tumor percent necrosis was unavailable in the final pathology report. Overall survival was determined by longevity from the time of surgery until the time of death, if applicable. The average age of our patient cohort was 22 years (range, 12-74 years) and the average followup was 3 years (range, 0.1-14 years). Full demographic variables for each of the three margin groups are provided (Table 3).

\section{Statistics}

Tumor percent necrosis (histological response), type of osteosarcoma, and amputation versus limb preservation were first investigated in their relation to margin status using univariate analysis. The chi-square test using the exact method with Monte Carlo estimation was performed for all variables. Time zero was determined to be the date of initial surgical resection. Local recurrence-free survival, metastases-free survival, and overall survival were calculated with the Kaplan-Meier method with the log-rank test. Cox proportional hazard models with forward selection at 
Table 4. Final multivariate models for local recurrence and overall survival

\begin{tabular}{llll}
\hline Variable & Level compared with reference & HR $(95 \%$ CI $)$ & p value \\
\hline Local recurrence & & & $1.799(0.3-10.786)$ \\
Margin grouped & Negative margins $\leq 1 \mathrm{~mm}$ & $8.006(1.314-48.781)$ & 0.5205 \\
Margin grouped & Positive margins & & 0.0241 \\
Overall survival & & $1.447(0.361-5.804)$ & 0.6024 \\
Margin grouped & Negative margins $\leq 1 \mathrm{~mm}$ & $6.26(1.499-26.142)$ & 0.0119 \\
Margin grouped & Positive margins &
\end{tabular}

$\mathrm{HR}=$ hazard ratio; $\mathrm{CI}$ = confidence interval.

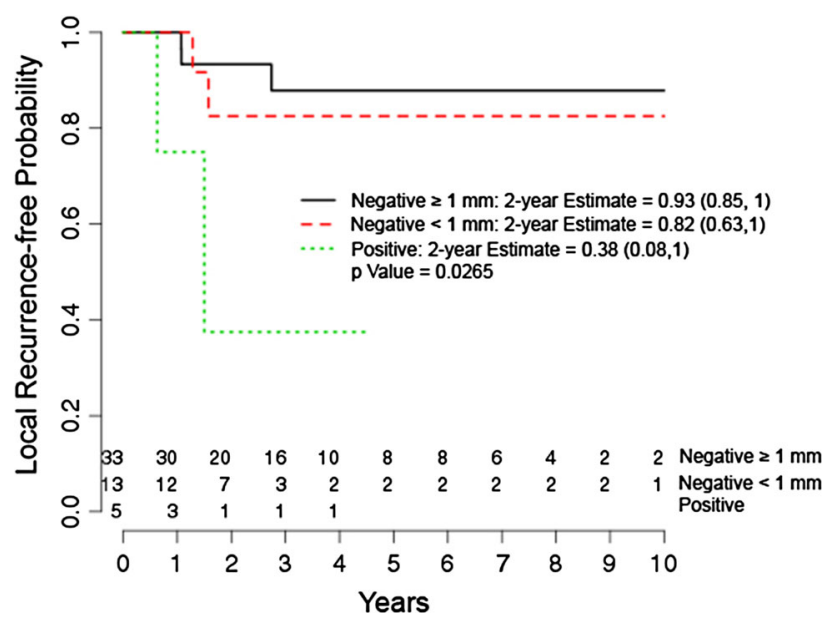

Fig. 2 The graph shows a Kaplan-Meier estimator curve for local recurrence by margin status.

inclusion $\alpha=0.05$ were used for multivariate analysis to identify factors predictive of local recurrence and overall survival. SAS software (SAS Institute, Inc, Cary, NC, USA) was used for all statistical analysis.

\section{Results}

\section{Local Recurrence}

After controlling for relevant confounding variables, the presence of a positive margin compared with a negative margin $>1 \mathrm{~mm}$ was the only independent predictor of local recurrence (hazard ratio, 8.006; 95\% confidence interval $[\mathrm{CI}], 1.314-48.781 ; \mathrm{p}=0.0241)$. Negative margins $\leq 1 \mathrm{~mm}$ did not show an increased likelihood of local recurrence in multivariate analysis with the numbers we had available for study (Table 4). Local recurrence overall was $14 \%$ (seven of 51) at an average of 3 years. Two-year local recurrence-free probability for margins $>1 \mathrm{~mm}$ was estimated to be $93 \%$ (95\% CI, $0.85-1.0$ ) versus $38 \%$ (95\% CI, 0.08-1.0) for positive margins (Fig. 2).

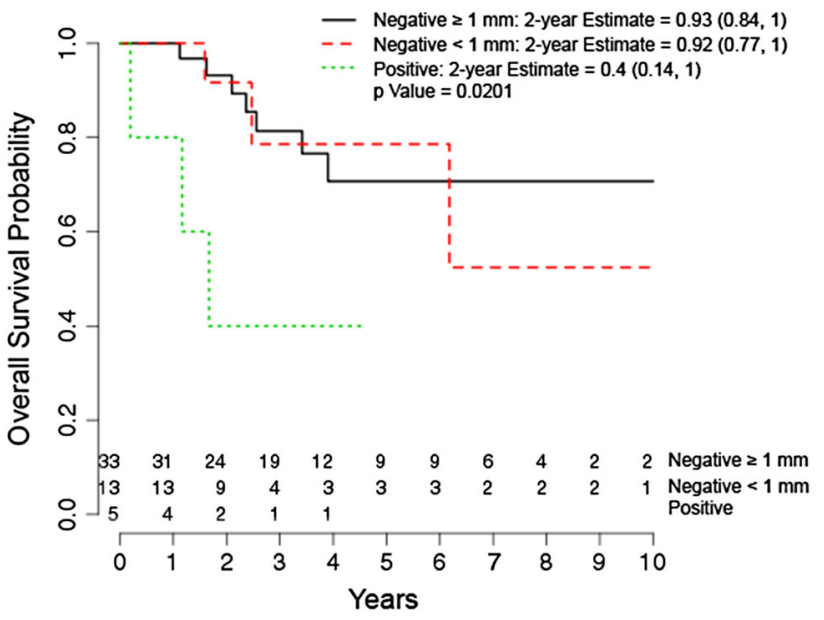

Fig. 3 The graph shows a Kaplan-Meier estimator curve of overall survival by margin status.

\section{Metastatic Disease}

With the numbers available, there was no difference in probability of developing metastatic disease among the three margin groups $(\mathrm{p}=0.614)$. The metastatic disease rate overall was $29 \%$ (15 of 51) at an average of 3.4 years.

\section{Overall Survival}

After adjusting for confounding variables, margin status was the only variable significantly associated with overall survival and was found to be a negative predictor (Table 4). Overall survival at an average of 4 years was $75 \%$ (38 of 51). Two-year overall survival probability was shown to be $93 \%$ (95\% CI, $0.84-1.0$ ) for negative margins $>1 \mathrm{~mm}$ versus $40 \%$ (95\% CI, 0.14-1.0) for positive margins (Fig. 3).

\section{Discussion}

Although it has long been believed that a positive margin portends a bad prognosis in osteosarcoma, the controversy 
about how much margin is necessary remains. In this study performed at a single institution allowing for consistency in both medical and surgical treatment as well as histological diagnosis of nonmetastatic osteosarcoma that originated in the long bone, we found that a positive margin portended an increased risk for local recurrence and overall worse survival and were able to identify this difference although we had relatively few patients in the series.

We recognize that there are several weaknesses to our study that limit the impact of the conclusions. First, it was a retrospective study not allowing us to capture all pertinent information but only what was listed in the medical records. We did have a significant portion of patients who were excluded from the study secondary to incomplete medical records relating to important endpoints to the study such as time after surgery to local recurrence as well as cause and date of death. If we were able to prospectively collect this information in a more complete manner this may have allowed for more patients to be included in the final cohort, thus potentially increasing the power of our study. Second, we did have a significant portion of patients lost to followup, excluding death, before 2 years. The patients lost to followup were not more likely to have positive margins in comparison to patients who had followup of a minimum of 2 years; however, the incidence of close margins $\leq 1 \mathrm{~mm}$ was slightly higher in the lost to followup group. One may argue that with the higher percentage of close margins lost to followup that maybe these patients had local recurrences or metastatic disease treated elsewhere. However, we did not find in our study that close negative margins were associated with worse probability for local recurrence, development of metastatic disease, or overall survival. We do admit that our short average followup of just over 3 years may not allow for the detection of local recurrence or metastatic disease that occurs later in the disease process. Third, the low incidence of osteosarcoma in the general patient population limits our study to small patient numbers, even over a 12 -year period. The low proportion of patients treated at our institution who met the inclusion criteria for our study, 51 out of a potential of 241 patients with osteosarcoma seen during the time period of the study, also introduces the potential for selection bias to our study. The early death of some patients may decrease the actual number of local recurrences because these patients did not survive long enough to go on to develop a local recurrence. We do concede that secondary to our low patient numbers, our study is likely underpowered. Thus, our findings of no difference in the development of metastatic disease across the three margin groups was likely underpowered because we did see a difference in overall survival and we know that patients die of metastatic disease with primary extremity osteosarcoma. Also, we were unable to show a significant effect from response to chemotherapy to either overall survival or local recurrence, both of which were affected by tumor percent necrosis in prior studies. This is likely secondary to our study being underpowered with exclusion of patients secondary to our strict inclusion criteria. Fourth, cause-specific mortality was not included because death certificates were not available for this study but only the date of death. This could potentially bias our overall survival results because patients could have died of other causes besides metastatic disease. This may help explain discrepancies seen in our data in which of five patients seen with positive margins, three died during the followup period but only two developed metastatic disease. Interestingly, in our 46 patients with negative margins, 13 developed metastases and 10 died during the followup period.

In our study, positive surgical margins were associated with a higher likelihood of local recurrence in multivariate analysis, which is in agreement with previous studies. $\mathrm{Li}$ et al. [11] showed that positive margins not only had a higher local recurrence rate, but was also a negative prognostic factor for local recurrence in multivariate analysis. However, in comparison to our patient cohort, this study included patients with osteosarcomas located in the pelvis and thorax, where positive margins can be more common secondary to anatomic constraints and complex anatomy. Bacci et al. [1] also noted that after multivariate analysis, surgical margins resulted in an independent prognostic factor for local recurrence. However, they noted that marginal margins were considered inadequate and at a higher risk of local recurrence. In contrast, our study showed that negative margins $\leq 1 \mathrm{~mm}$, which could be considered a marginal margin, did not show an increased likelihood of local recurrence compared with margins greater than $1 \mathrm{~mm}$ with the numbers we had available for study. This is supported by Li et al. [11] in which close margins (5 $\mathrm{mm}$ or less) compared with wider margins (greater than $5 \mathrm{~mm}$ ) did not increase the likelihood of local recurrence as well as by Bispo et al. [3] where no difference in local recurrence was detected between margins less than $2 \mathrm{~mm}$ and margins greater than $2 \mathrm{~mm}$.

There was no association between margin status and the development of distant metastatic disease after surgical resection with the numbers we had available for our study. There is little in the way of prior research evaluating this exact relationship. Grimer et al. [7] looked at 202 patients and found no prognostic factors for the development of metastatic disease. The overall rate of metastatic disease in that study was $50 \%$ at a mean of 15 months after diagnosis. Kim et al. [10] developed a nomogram to predict the probability for the development of metastatic disease in patients with Stage IIB osteosarcoma including such variables as tumor percent necrosis, location, presence of intraor extraarticular joint extension, and alkaline phosphatase 
level at diagnosis. They did not include margin status and stated that the association between incomplete surgical resections and the development of metastatic disease has not been proven. However, as mentioned in our limitations, more patient numbers are needed to fully elucidate this relationship because we were able to show a decreased overall survival with positive margins and patients die of metastatic disease with primary extremity osteosarcoma.

Our study showed that patients with a positive margin had a worse overall survival probability at 5 years compared with those with negative margins and that, after adjusting for confounding variables, positive margins were an independent prognostic factor in predicting overall survival. Whether surgical margin correlates with overall survival in patients with osteosarcoma is controversial. Inadequate surgical margins have been found to be associated with overall worse event-free survival in extremity osteosarcomas but were not associated with decreased overall survival [11]. Other studies have also found no association between surgical margins and overall survival. Ford et al. [5] looked at a total of 428 patients across two large orthopaedic oncology centers and found that adequacy of surgical margins was not associated with overall survival. However, margins were graded qualitatively (intralesional, marginal, wide, and radical) versus quantitatively and the length of followup was not recorded. Thus, it is possible that patients with positive or close margins who developed local recurrence were treated elsewhere thereby not allowing for a relationship between surgical margin and overall survival to be identified. The literature is scant of the presence of microscopic positive margins, which were all positive margins in our series, and its relationship to overall survival. However, incomplete surgical resection with retained macroscopic disease showed a $15 \%$ overall survival rate at 5 years with a relative risk of overall survival of 3.60 when compared with complete surgical resection with negative margins [2].

In conclusion, we found that patients with positive margins in extremity, nonmetastatic, high-grade osteosarcoma were more likely to develop a local recurrence and had a worse overall survival predicted at 2 years than did patients with negative margins. We could not show a relationship between margin and metastatic rate, which is counterintuitive and likely relates to insufficient statistical power related to our small sample size. Further studies with more numbers of patients are necessary to confirm these preliminary observations. Even so, we believe surgeons should strive to obtain negative margins while attempting to maximize function and quality of life.

Acknowledgments We thank William Fulp for his assistance with the statistical analysis for this research. We also thank the attending pathologists over the time period of the study including Carlos Muro Cacho MD, and Marilyn Bui MD, PhD.

\section{References}

1. Bacci G, Longhi A, Versari M, Mercuri M, Briccoli A, Picci P. Prognostic factors for osteosarcoma of the extremity treated with neoadjuvant chemotherapy. Cancer. 2006;106:1154-1161.

2. Bielack SS, Kempf-Bielack B, Delling G, Exner GU, Flege S, Helmke K, Kotz R, Salzer-Kuntschik M, Werner M, Winkelmann W, Zoubek A, Jurgens H, Winkler K. Prognostic factors in highgrade osteosarcoma of the extremities or trunk: an analysis of 1,702 patients treated on neoadjuvant cooperative osteosarcoma study group protocols. J Clin Oncol. 2002;20:776-790.

3. Bispo RZ Jr, de Camargo OP. Prognostic factors in the survival of patients diagnosed with primary non-metastatic osteosarcoma with a poor response to neoadjuvant chemotherapy. Clinics. 2009;64:1177-1186.

4. Carrle D, Bielack SS. Current strategies of chemotherapy in osteosarcoma. Int Orthop. 2006;30:445-451.

5. Ford S, Saithna A, Grimer RJ, Picci P. Comparison of the outcome of conventional osteosarcoma at two specialist international orthopaedic oncology centres. Sarcoma. 2004;8:13-18.

6. Goorin AM, Schwartzentruber DJ, Devidas M, Gebhardt MC, Ayala AG, Harris MB, Helman LJ, Grier HE, Link MP; Pediatric Oncology Group. Presurgical chemotherapy compared with immediate surgery and adjuvant chemotherapy for nonmetastatic osteosarcoma: Pediatric Oncology Group Study POG-8651. J Clin Oncol. 2003;21:1574-1580.

7. Grimer RJ, Taminiau AM, Cannon SR. Surgical outcomes in osteosarcoma. J Bone Joint Surg Br. 2002;84:395-400.

8. Huvos A. Osteosarcoma: Pathologic Assessment of Preoperative (neoadjuvant) Chemotherapy in Bone Tumors: Diagnosis, Treatment, and Prognosis. $2^{\text {nd }}$ ed. Philadelphia, PA, USA: WB Saunders; 1991:122-128.

9. Kawaguchi N, Ahmed AR, Matsumoto S, Manabe J, Matsushita Y. The concept of curative margin in surgery for bone and soft tissue sarcoma. Clin Orthop Relat Res. 2004;419:165-172.

10. Kim SH, Shin K, Kim HY, Cho YJ, Noh JK, Suh JS, Yang WI. Postoperative nomogram to predict the probability of metastasis in Enneking stage IIB extremity osteosarcoma. BMC Cancer. 2014;12:666.

11. Li X, Moretti V, Ashana A, Lackman R. Impact of close surgical margin on local recurrence and survival in osteosarcoma. Int Orthop. 2012;36:131-137. 